\title{
Quantitative assessment of polymorphisms in H19 IncRNA and cancer risk: a meta-analysis of 13,392 cases and 18,893 controls
}

\author{
Minjie Chu ${ }^{1, *}$, Weiyan Yuan ${ }^{2, *}$, Shuangshuang $\mathbf{W u}^{3, *}$, Zhiquan Wang ${ }^{4, *}$, Liping Mao ${ }^{5}$, \\ Tian Tian ${ }^{1}$, Yihua Lu${ }^{1}$, Bowen Zhu ${ }^{1}$, Yue Yang${ }^{1}$, Bin Wang ${ }^{6}$, Haiquan Gao ${ }^{6}$, Liying \\ Jiang ${ }^{1, \#}$, Xun Zhuang ${ }^{1, \#}$ \\ ${ }^{1}$ Department of Epidemiology and Biostatistics, School of Public Health, Nantong University, Nantong, Jiangsu, China \\ ${ }^{2}$ Department of Gastroenterology, Affiliated Hospital of Nantong University, Nantong, Jiangsu, China \\ ${ }^{3}$ Department of Geriatrics, The First Affiliated Hospital of Nanjing Medical University, Nanjing, Jiangsu, China \\ ${ }^{4}$ Center for Disease Control and Prevention of Nantong, Nantong, Jiangsu, China \\ ${ }^{5}$ Department of Oncology, the Sixth People's Hospital of Nantong, Nantong, Jiangsu, China \\ ${ }^{6}$ Nantong Prison Hospital, Nantong, Jiangsu, China \\ *These authors contributed equally to this work \\ \#These authors jointly directed to this work
}

Correspondence to: Minjie Chu, email: chuminjie@ntu.edu.cn

Keywords: H19, polymorphism, cancer and meta-analysis

Received: June 13, 2016

Accepted: October 03, 2016

Published: October 08, 2016

\section{ABSTRACT}

H19 refers to a long non-coding RNA (IncRNA) that functions as an oncogenic molecule in different cancer cells. Genetic variants of $\mathrm{H19}$ may affect the activity of certain regulatory factors, which subsequently regulate the aberrant expression of H19. This feedback loop might be one of the underlying mechanisms influencing tumour susceptibility and prognosis. Although there have been several recent studies that examined possible links between polymorphisms in $\mathrm{H19}$ and cancer risk, the results have been inconclusive. Thus, we performed a meta-analysis to estimate the associations between $\mathrm{H19}$ polymorphisms (rs2107425, rs2839698 and rs217727) and cancer risk. Ten studies comprising 13,392 cases and 18,893 controls were included in the study. Overall, the variant $\mathrm{T}$ allele of rs 2107425 correlated with a significantly decreased risk of developing cancer (dominant model: $\mathrm{OR}=0.86 ; 95 \% \mathrm{CI}=0.76-0.98$ ). In addition, a marginally significant association between the rs 2839698 and cancer risk was observed (dominant model: $O R=1.09 ; 95 \% C I=0.99-1.20$ ). After stratification for ethnicity, it became apparent that Asians with the variant A allele of rs2839698 exhibited a significantly higher risk of developing cancer (dominant model: $O R=1.11 ; 95 \% C I=1.01-1.23)$. Interestingly, the rs2839698 variant was also significant associated with an increased risk of cancers of the digestive system (dominant model: $O R=1.23 ; 95 \% \mathrm{CI}=1.08-1.41$ ). These findings provided evidence that $\mathrm{H19}$ rs2107425 may modify general cancer susceptibility, while rs2839698 may modify cancer susceptibility based on ethnicity and type. Further experimental studies to evaluate the limits of this hypothesis are warranted, and future functional studies are required to clarify the possible mechanisms.

\section{INTRODUCTION}

Cancer has long been a major public health problem. Currently, it is the leading cause of morbidity and mortality worldwide. In 2016, it is estimated that 1,685,210 new cancer cases and 595,690 cancer deaths will occur in the United States [1], while an estimated 4,292,000 new cancer cases and 2,814,000 cancer deaths were projected in China in 2015 [2]. Various factors contribute to cancer's development, with environmental and genetic factors being the most common. In particular, the emergence of highthroughput RNA sequencing (RNA-Seq) technologies 
have provided a revolutionary means for systematically analysing the role of non-coding genomic transcripts in regulating gene expression, and by extension their impact on disease development and progression. Among these transcripts, long non-coding RNAs (lncRNAs) are emerging as significant regulators in tumourigenesis and progressions [3, 4], and numerous IncRNAs have been identified in multiple cancer transcriptomes [5]. One such lncRNA is the imprinted maternally expressed, nonprotein coding transcript $H 19$. The relationship between an aberrant expression of $H 19$ and cancer prognosis has been explored by many researchers. Furthermore, a recent metaanalyses performed by Chen et al. has demonstrated that high levels of $H 19$ expression may serve as a predictive indicator of poor prognoses in multiple cancers. Metaanalysis has also revealed that a high expression of $H 19$ is significantly related to lymph node metastasis, another influence on cancer prognoses [6].

Recently, genome-wide association study (GWAS) has identified a significant association between the single nucleotide polymorphism (SNP) rs2107425 and breast cancer risk [7]. SNP rs2107425 is located approximately $2 \mathrm{~kb}$ upstream of H19 lncRNA. In 2012, Riaz et al. analysed mRNA expression of the gene $H 19$ most likely to be closely located to SNP rs2107425 in a subset of 1,401 primary breast tumours. However, no significant difference between the respective three genotype groups of rs2107425 and $H 19$ mRNA expression was observed [8]. These results indicate that the SNP rs2107425 is unlikely itself to be a causative agent of breast cancer and that a more thorough evaluation of variations in the associated $H 19$ gene region is warranted. As expected, the associations of $H 19$ polymorphisms with cancer sensitivities have attracted much interest, with particular focus on $H 19$ rs2839698 and rs217727. Based on the Encyclopedia of DNA Elements (ENCODE) DNase I hypersensitive site (DHS) sequencing data set, we found the two H19 SNPs (rs2839698 and rs217727) are within open chromatin regions associated with gene regulatory elements, indicating that both of the SNPs may affect the binding of transcription factors. ChIP-Seq data from the ENCODE project further demonstrates that rs 217727 is located in a region that may influence the binding of numerous transcription factors. These results show that it is biologically conceivable for the SNPs (rs2839698 and rs217727) in $H 19$ to be potential causal variants that regulate the expression of $\mathrm{H} 19$ and further affect cancer development and progression.

As expected, the association of two SNPs (rs2839698 and rs217727) together with rs2107425 in H19 with cancers susceptibility has attracted much interest in subsequent research [9-18]. Unfortunately, the research exploring this association has not been able to reach a consensus. For instance, a previous study reported that the variant genotype of rs2839698 was definitely associated with increased risk for colorectal and gastric cancers in the Chinese population $[9,11]$. However, the rs 2839698 polymorphism exhibited the opposite associations for cancer risk in Caucasians (a population in the Netherlands) [14]. Thus, we performed a meta-analysis using currently published data to more precisely characterize the associations of rs2107425, rs2839698 and rs217727 in $H 19$ lncRNA with cancer risks.

\section{RESULTS}

\section{Characteristics of the published studies}

Following the application of strict screening criteria, 10 articles evaluating a total of 13,392 cases and 18,893 controls concerning gastric, bladder, colorectal, breast, ovarian and lung cancers were ultimately included in our quantitative analysis (Figure 1). The general characteristics of the included studies are listed in Table 1. Among these studies, five studies were carried out among Asian populations and five studies were carried out among Caucasian populations. Three articles reported the effects of $H 19$ polymorphisms in breast cancer, two reported in bladder cancer, two in ovarian cancer, one in gastric cancer, one in lung cancer and one in colorectal cancer. Among the studies that explored the relationships between H19 SNPs with cancer risk, one focused on 3 SNPs (rs2107425, rs2839698 and rs217727), and four focused on 2 SNPs, while the other five focused on only one SNP. Genotyping was performed using TaqMan in 5 studies, Sequenom in 3 studies, created restriction site PCR (CRS-RFLP) in 1 study, GoldenGate assay in 1 study and PCR-RFLP in 1 study. In addition, there was no evidence to prove that genotype frequencies among the controls deviated from those expected under the Hardy-Weinberg equilibrium (HWE) for each SNP studied. The distributions of genotypes and alleles of $H 19$ polymorphisms (rs2107425, rs2839698 and rs217727) for each individual study are listed in Supplementary Tables S2-S4.

\section{Quantitative synthesis}

Evaluations of the associations of rs 2107425 with cancer risks are presented in Table 2. The variant $\mathrm{T}$ allele of rs 2107425 was correlated with a significantly decreased risk of developing cancer (dominant model: $\mathrm{OR}=0.86$; $95 \% \mathrm{CI}=0.76-0.98, P=0.005$ for the heterogeneity test, $I^{2}=70.3 \%$; Figure 2). The results of other tested models are listed in Table 2. Next, we evaluated the effect of the rs 2107425 polymorphism on cancer risk among the subgroups (Table 2). In the stratified analyses, associations between rs2107425 and cancer risk were still significant among Caucasians (dominant model: $\mathrm{OR}=0.84 ; 95 \%$ $\mathrm{CI}=0.74-0.97 ; P=0.003$ for the heterogeneity test, $I^{2}=75.0 \%$ ), in studies with population-based controls (dominant model: $\mathrm{OR}=0.84 ; 95 \% \mathrm{CI}=0.74-0.97$; 
Table 1: Characteristics of the studies included in the meta-analysis

\begin{tabular}{|c|c|c|c|c|c|c|c|c|}
\hline First Author & Year & Country & Ethnicity & Type of cancer & $\begin{array}{c}\text { Case/ } \\
\text { Control }\end{array}$ & Source of controls & Platform & $\begin{array}{l}\text { Genotyped } \\
\text { SNPs }\end{array}$ \\
\hline Hua & 2016 & China & Asian & bladder cancer & $1049 / 1399$ & Hospital-based & TaqMan & $\begin{array}{l}\text { rs } 217727, \\
\text { rs2839698 }\end{array}$ \\
\hline $\mathrm{Li}$ & 2016 & China & Asian & colorectal cancer & $1147 / 1203$ & Population-based & TaqMan & $\begin{array}{l}\text { rs } 217727, \\
\text { rs2839698 }\end{array}$ \\
\hline $\mathrm{Xia}$ & 2016 & China & Asian & breast cancer & $464 / 467$ & Population-based & CRS-RFLP ${ }^{a}$ & rs 217727 \\
\hline Gong & 2016 & China & Asian & lung cancer & $498 / 213$ & Hospital-based & Sequenom & $\begin{array}{l}\text { rs } 2839698, \\
\text { rs2107425 }\end{array}$ \\
\hline Yang & 2015 & China & Asian & gastric cancer & $500 / 500$ & Hospital-based & TaqMan & $\begin{array}{l}\text { rs } 217727, \\
\text { rs2839698 }\end{array}$ \\
\hline Butt & 2012 & Sweden & Caucasian & breast cancer & $728 / 1448$ & Population-based & Sequenom & rs2107425 \\
\hline Barnholtz-Sloan & 2010 & USA & Caucasian & breast cancer & $1972 / 1776$ & Population-based & $\begin{array}{l}\text { GoldenGate } \\
\text { assay }\end{array}$ & rs 2107425 \\
\hline Quaye & 2009 & Multinational $\mathrm{l}^{\mathrm{b}}$ & Caucasian & ovarian cancer & $1491 / 3145$ & Population-based & Taqman & rs2107425 \\
\hline Song & 2009 & Multinational $^{\mathrm{c}}$ & Caucasian & ovarian cancer & $5366 / 8538$ & Population-based & $\begin{array}{l}\text { Taqman }+ \\
\text { Sequenom }\end{array}$ & rs 2107425 \\
\hline Verhaegh & 2008 & $\begin{array}{l}\text { The } \\
\text { Netherlands }\end{array}$ & Caucasian & bladder cancer & $177 / 204$ & Population-based & PCR-RFLP & $\begin{array}{l}\text { rs217727, } \\
\text { rs2839698, } \\
\text { rs2107425 }\end{array}$ \\
\hline
\end{tabular}

${ }^{\mathrm{a}}$ created restriction site PCR.

bincluding UK, Denmark and USA.

cincluding European countries, USA and Australia.

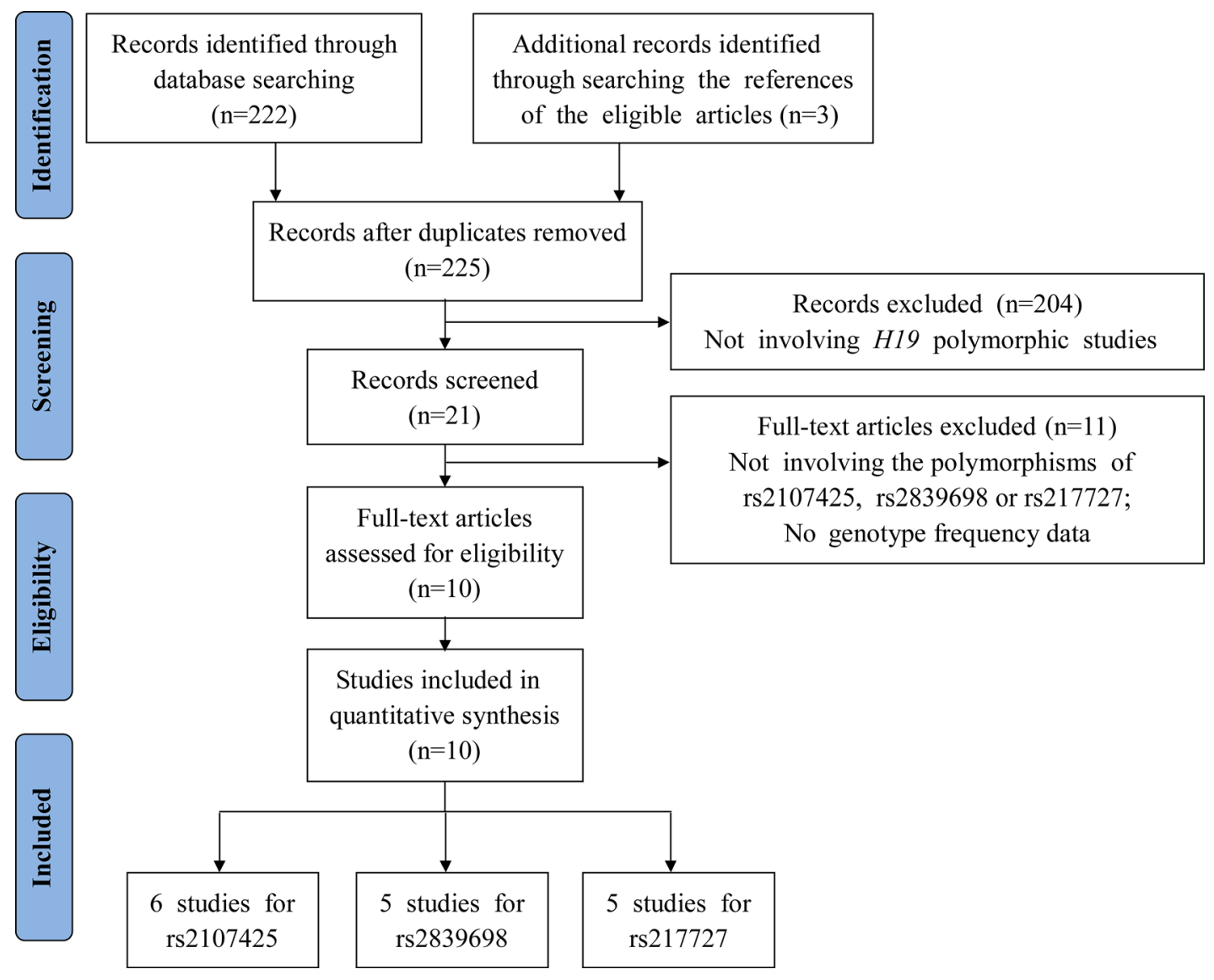

Figure 1: Flow diagram of the study selection process. 
Table 2: Summary ORs of the H19 rs2107425 polymorphism and cancer risk

\begin{tabular}{|c|c|c|c|c|c|c|c|c|c|c|}
\hline \multirow{2}{*}{ Variables } & \multirow{2}{*}{ Studies } & \multicolumn{3}{|c|}{ CT versus $\mathbf{C C}$} & \multicolumn{3}{|c|}{ TT versus $\mathbf{C C}$} & \multicolumn{3}{|c|}{ Dominant model } \\
\hline & & OR(95\%CI) & $P^{a}$ & $I^{2}$ & OR $(95 \% C I)$ & $P^{a}$ & $I^{2}$ & OR(95\%CI) & $P^{a}$ & $I^{2}$ \\
\hline Total & 6 & $0.84(0.73-0.97)$ & 0.002 & $73.6 \%$ & $0.97(0.89-1.06)$ & 0.798 & $0.0 \%$ & $0.86(0.76-0.98)$ & 0.005 & $70.3 \%$ \\
\hline \multicolumn{11}{|l|}{ Ethnicity } \\
\hline Asians & 1 & $1.07(0.75-1.52)$ & & & $0.98(0.59-1.65)$ & & & $1.05(0.75-1.47)$ & & \\
\hline Caucasians & 5 & $0.82(0.71-0.96)$ & 0.001 & $77.5 \%$ & $0.97(0.89-1.06)$ & 0.671 & $0.0 \%$ & $0.84(0.74-0.97)$ & 0.003 & $75.0 \%$ \\
\hline \multicolumn{11}{|l|}{ Cancer type } \\
\hline breast cancer & 2 & $0.84(0.58-1.23)$ & 0.014 & $83.4 \%$ & $0.94(0.79-1.12)$ & 0.329 & $0.0 \%$ & $0.85(0.60-1.20)$ & 0.016 & $82.6 \%$ \\
\hline ovarian cancer & 2 & $0.82(0.65-1.04)$ & 0.002 & $89.2 \%$ & $0.98(0.89-1.09)$ & 0.268 & $18.4 \%$ & $0.84(0.68-1.05)$ & 0.003 & $88.6 \%$ \\
\hline lung cancer & 1 & $1.07(0.75-1.52)$ & & & $0.98(0.59-1.65)$ & & & $1.05(0.75-1.47)$ & & \\
\hline bladder cancer & 1 & $0.66(0.43-1.01)$ & & & $1.02(0.51-2.03)$ & & & $0.72(0.48-1.07)$ & & \\
\hline \multicolumn{11}{|l|}{ Source of controls } \\
\hline Population-based & 5 & $0.82(0.71-0.96)$ & 0.001 & $77.5 \%$ & $0.97(0.89-1.06)$ & 0.671 & $0.0 \%$ & $0.84(0.74-0.97)$ & 0.003 & $75.0 \%$ \\
\hline Hospital-based & 1 & $1.07(0.75-1.52)$ & & & $0.98(0.59-1.65)$ & & & $1.05(0.75-1.47)$ & & \\
\hline \multicolumn{11}{|l|}{ Case sample size } \\
\hline$\geq 500$ & 4 & $0.84(0.72-0.98)$ & 0.001 & $81.2 \%$ & $0.97(0.89-1.06)$ & 0.506 & $0.0 \%$ & $0.86(0.74-0.99)$ & 0.002 & $79.7 \%$ \\
\hline$<500$ & 2 & $0.88(0.67-1.15)$ & 0.085 & $66.30 \%$ & $0.99(0.66-1.51)$ & 0.934 & $0.00 \%$ & $0.90(0.69-1.16)$ & 0.154 & $50.90 \%$ \\
\hline
\end{tabular}

a $P$ for heterogeneity (a random-effects model was used when the $P$ value for heterogeneity test was $<0.05$; otherwise, a fixed-effect model was used).

$P=0.003$ for the heterogeneity test, $\left.I^{2}=75.0 \%\right)$ and in studies with case sample size $\geq 500$ (dominant model: $\mathrm{OR}=0.86 ; 95 \% \mathrm{CI}=0.74-0.99 ; P=0.002$ for the heterogeneity test, $I^{2}=79.7 \%$ ).

The evaluations of the associations of rs 2839698 with cancer risks are presented in Table 3. The variant A allele exhibited a marginally significant association with cancer risk in the dominant model $(\mathrm{OR}=1.09 ; 95 \%$ $\mathrm{CI}=0.99-1.20, P=0.113$ for the heterogeneity test, $I^{2}=46.5 \%$; Figure 3 ). The results of other tested models are listed in Table 3.

Next, we evaluated the effect of the rs2839698 polymorphism on cancer risk among the subgroups (Table 3). In the stratified analyses, the rs2839698 SNP had significant association with increased cancer risk among Asians (Chinese; dominant model: OR = 1.11; $95 \% \mathrm{CI}=1.01-1.23 ; P=0.158$ for the heterogeneity test, $I^{2}=42.3 \%$ ). Beyond that, the rs2839698 variant exhibited a significant association with an increased risk of digestive system cancers (dominant model: $\mathrm{OR}=1.23$; $95 \% \mathrm{CI}=1.08-1.41 ; P=0.546$ for the heterogeneity test, $I^{2}=0.0 \%$ ). Interestingly, the variant A allele of rs 2839698 was significantly associated with an increased risks of developing cancer among studies with a case sample size $\geq 500$ (dominant model: $\mathrm{OR}=1.12 ; 95 \% \mathrm{CI}=1.01-1.24$; $P=0.089$ for the heterogeneity test, $I^{2}=58.7 \%$ ).

The evaluations of the associations between SNP rs 217727 and cancer risk are displayed in Figure 4. Overall, the A variant allele of rs217727 exhibited no significant association with cancer risks (dominant model: $\mathrm{OR}=0.94$;

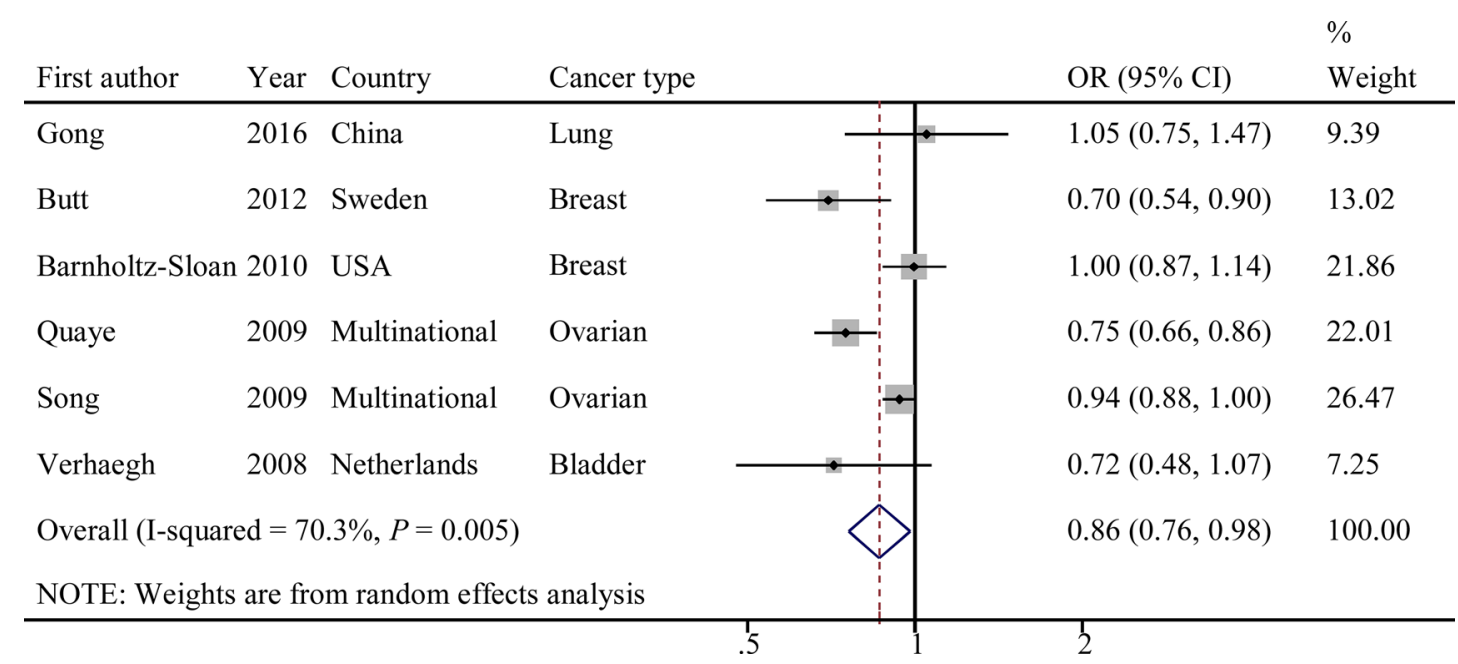

Figure 2: Forest plot of $\mathrm{OR}$ with $95 \% \mathrm{CI}$ for the $\mathrm{H19}$ rs2107425 with cancer risk under dominant model. 
Table 3: Summary ORs of the H19 rs2839698 polymorphism and cancer risk

\begin{tabular}{|c|c|c|c|c|c|c|c|c|c|c|}
\hline \multirow{2}{*}{ Variables } & \multirow{2}{*}{ Studies } & \multicolumn{3}{|c|}{ GA versus GG } & \multicolumn{3}{|c|}{ AA versus GG } & \multicolumn{3}{|c|}{ Dominant model } \\
\hline & & OR(95\%CI) & $P^{a}$ & $I^{2}$ & OR $(95 \% C I)$ & $P^{a}$ & $I^{2}$ & OR(95\%CI) & $P^{\mathrm{a}}$ & $I^{2}$ \\
\hline Total & 5 & $1.07(0.96-1.18)$ & 0.130 & $43.8 \%$ & $1.15(0.83-1.58)$ & 0.019 & $66.1 \%$ & $1.09(0.99-1.20)$ & 0.113 & $46.5 \%$ \\
\hline \multicolumn{11}{|l|}{ Ethnicity } \\
\hline Asians & 4 & $1.09(0.98-1.21)$ & 0.396 & $0.0 \%$ & $1.15(0.78-1.69)$ & 0.008 & $74.4 \%$ & $1.11(1.01-1.23)$ & 0.158 & $42.3 \%$ \\
\hline Caucasians & 1 & $0.65(0.40-1.06)$ & & & $1.10(0.63-1.92)$ & & & $0.78(0.50-1.22)$ & & \\
\hline \multicolumn{11}{|l|}{ Cancer type } \\
\hline digestive system cancer ${ }^{b}$ & 2 & $1.17(1.02-1.35)$ & 0.594 & $0.0 \%$ & $1.58(1.22-2.05)$ & 0.840 & $0.0 \%$ & $1.23(1.08-1.41)$ & 0.546 & $0.0 \%$ \\
\hline bladder cancer & 2 & $0.94(0.80-1.10)$ & 0.123 & $58.0 \%$ & $1.03(0.79-1.36)$ & 0.807 & $0.0 \%$ & $0.96(0.82-1.11)$ & 0.340 & $0.0 \%$ \\
\hline lung cancer & 1 & $1.15(0.81-1.63)$ & & & $0.60(0.35-1.04)$ & & & $1.01(0.73-1.40)$ & & \\
\hline \multicolumn{11}{|l|}{ Source of controls } \\
\hline Population-based & 2 & $0.91(0.53-1.55)$ & 0.032 & $78.2 \%$ & $1.43(1.08-1.88)$ & 0.290 & $10.9 \%$ & $1.14(0.98-1.33)$ & 0.076 & $68.2 \%$ \\
\hline Hospital-based & 3 & $1.06(0.93-1.21)$ & 0.284 & $20.6 \%$ & $1.02(0.62-1.67)$ & 0.019 & $74.7 \%$ & $1.06(0.94-1.20)$ & 0.149 & $47.6 \%$ \\
\hline \multicolumn{11}{|l|}{ Case sample size } \\
\hline$\geq 500$ & 3 & $1.09(0.97-1.21)$ & 0.237 & $30.5 \%$ & $1.32(1.08-1.61)$ & 0.096 & $57.3 \%$ & $1.12(1.01-1.24)$ & 0.089 & $58.7 \%$ \\
\hline$<500$ & 2 & $0.95(0.72-1.25)$ & 0.063 & $71.1 \%$ & $0.81(0.55-1.20)$ & 0.133 & $55.7 \%$ & $0.92(0.71-1.20)$ & 0.357 & $0.0 \%$ \\
\hline
\end{tabular}

$95 \% \mathrm{CI}=0.78-1.12, P=0.018$ for the heterogeneity test, $I^{2}=66.3 \%$ ). The results of other tested models are listed in Table 4. In further stratified analyses, rs217727 SNP was significantly associated with decreased cancer risk among studies with population-based controls (dominant model: $\mathrm{OR}=0.82 ; 95 \% \mathrm{CI}=0.72-0.94 ; P=0.739$ for the heterogeneity test, $I^{2}=0.0 \%$ ). Additionally, the variant A allele of rs217727 was significantly associated with a decreased risk of developing cancer among studies with a case sample size $<500$ (dominant model: $\mathrm{OR}=0.78$; $95 \% \mathrm{CI}=0.62-0.98 ; P=0.636$ for the heterogeneity test, $I^{2}=0.0 \%$; Table 4).

\section{Test of heterogeneity}

For rs217727, significant heterogeneity was observed after the data were pooled (dominant model: $P$ for heterogeneity $=0.018, I^{2}=66.3 \%$ ). As shown in Table 4, when the subjects were stratified on the basis of ethnicity, heterogeneity remained in Asians (dominant model: $P$ for heterogeneity $=0.018, I^{2}=70.3 \%$ ). Additionally, in analyses of control sources, the heterogeneity disappeared in studies with population-based controls (dominant model: $P$ for heterogeneity $=0.739, I^{2}=0.0 \%$ ), as well as in hospital-based controls (dominant model: $P$ for heterogeneity $=0.061, I^{2}=71.6 \%$ ). Furthermore, heterogeneity disappeared in studies with a case sample size $<500$ (dominant model: $P$ for heterogeneity $=0.636$, $I^{2}=0.0 \%$ ). Nonetheless, heterogeneity was still present in studies with a case sample size $\geq 500$ (dominant model: $P$ for heterogeneity $=0.015, I^{2}=76.2 \%$ ).

\section{Sensitivity analysis}

To test the stability of the rs217727 results, we conducted sensitivity analyses by sequentially removing each

\begin{tabular}{|c|c|c|c|c|c|c|}
\hline First author & Year & Country & Cancer type & & OR $(95 \% \mathrm{CI})$ & $\begin{array}{l}\% \\
\text { Weight }\end{array}$ \\
\hline Hua & 2016 & China & Bladder & $\longrightarrow$ & $0.98(0.84,1.15)$ & 38.10 \\
\hline $\mathrm{Li}$ & 2016 & China & Colorectal & 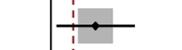 & $1.20(1.02,1.41)$ & 33.67 \\
\hline Gong & 2016 & China & Lung & 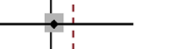 & $1.01(0.73,1.40)$ & 9.13 \\
\hline Yang & 2015 & China & Gastric & $\rightarrow$ & $1.31(1.02,1.69)$ & 13.65 \\
\hline Verhaegh & 2008 & Netherlands & Bladder $\leftarrow$ & 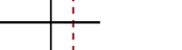 & $0.78(0.50,1.22)$ & 5.45 \\
\hline \multicolumn{4}{|c|}{ Overall $($ I-squared $=46.5 \%, P=0.113)$} & 引〉 & $1.09(0.99,1.20)$ & 100.00 \\
\hline
\end{tabular}

Figure 3: Forest plot of OR with $95 \%$ CI for the $H 19$ rs2839698 with cancer risk under dominant model. 
Table 4: Summary ORs of the H19 rs217727 polymorphism and cancer risk

\begin{tabular}{|c|c|c|c|c|c|c|c|c|c|c|}
\hline \multirow{2}{*}{ Variables } & \multirow{2}{*}{ Studies } & \multicolumn{3}{|c|}{ GA versus GG } & \multicolumn{3}{|c|}{ AA versus GG } & \multicolumn{3}{|c|}{ Dominant model } \\
\hline & & OR $(95 \% C I)$ & $P^{a}$ & $I^{2}$ & OR $(95 \% C I)$ & $P^{\mathrm{a}}$ & $I^{2}$ & OR $(95 \% C I)$ & $P^{\mathbf{a}}$ & $I^{2}$ \\
\hline Total & 5 & $0.88(0.73-1.06)$ & 0.022 & $65.0 \%$ & $1.10(0.83-1.48)$ & 0.011 & $69.5 \%$ & $0.94(0.78-1.12)$ & 0.018 & $66.3 \%$ \\
\hline \multicolumn{11}{|l|}{ Ethnicity } \\
\hline Asians & 4 & $0.90(0.73-1.11)$ & 0.014 & $71.9 \%$ & $1.16(0.87-1.54)$ & 0.012 & $72.7 \%$ & $0.97(0.80-1.17)$ & 0.018 & $70.3 \%$ \\
\hline Caucasians & 1 & $0.74(0.49-1.14)$ & & & $0.45(0.13-1.50)$ & & & $0.71(0.47-1.08)$ & & \\
\hline \multicolumn{11}{|l|}{ Cancer type } \\
\hline digestive system cancer ${ }^{b}$ & 2 & $1.02(0.71-1.47)$ & 0.024 & $80.4 \%$ & $1.16(0.57-2.34)$ & 0.002 & $89.3 \%$ & $1.05(0.67-1.64)$ & 0.004 & $88.0 \%$ \\
\hline bladder cancer & 2 & $0.90(0.77-1.06)$ & 0.331 & $0.0 \%$ & $1.20(0.93-1.54)$ & 0.100 & $63.0 \%$ & $0.95(0.82-1.11)$ & 0.143 & $53.5 \%$ \\
\hline breast cancer & 1 & $0.64(0.47-0.87)$ & & & $1.11(0.79-1.55)$ & & & $0.81(0.61-1.06)$ & & \\
\hline \multicolumn{11}{|l|}{ Source of controls } \\
\hline Population-based & 3 & $0.79(0.69-0.91)$ & 0.256 & $26.6 \%$ & $0.90(0.74-1.09)$ & 0.192 & $39.4 \%$ & $0.82(0.72-0.94)$ & 0.739 & $0.0 \%$ \\
\hline Hospital-based & 2 & $1.01(0.88-1.17)$ & 0.080 & $67.3 \%$ & $1.38(1.11-1.71)$ & 0.221 & $33.3 \%$ & $1.08(0.94-1.24)$ & 0.061 & $71.6 \%$ \\
\hline \multicolumn{11}{|l|}{ Case sample size } \\
\hline$\geq 500$ & 3 & $0.95(0.85-1.06)$ & 0.076 & $61.1 \%$ & $1.18(0.80-1.75)$ & 0.004 & $81.8 \%$ & $1.02(0.81-1.28)$ & 0.015 & $76.2 \%$ \\
\hline$<500$ & 2 & $0.67(0.53-0.86)$ & 0.571 & $0.0 \%$ & $1.03(0.75-1.42)$ & 0.156 & $50.30 \%$ & $0.78(0.62-0.98)$ & 0.636 & $0.0 \%$ \\
\hline
\end{tabular}

${ }^{\mathrm{a}} P$ for heterogeneity (a random-effects model was used when the $P$ value for heterogeneity test was $<0.05$; otherwise, a fixed-effect model was used).

eligible study (Supplementary Table S5). A study by Yang et al. that focused on gastric cancer was the major contributor of heterogeneity in the dominant model $\left(I^{2}=66.3 \%\right.$, $P$ for heterogeneity $=0.018)$. After removing this study, heterogeneity was significantly reduced $\left(I^{2}=19.90 \%, P\right.$ for heterogeneity $=0.290)$. As expected, similar results were observed in other genetic models (i.e., GA versus GG and AA versus GG), indicating that Yang et al.'s study on gastric cancer markedly changed the pooled OR.

\section{Publication bias}

We utilized funnel plots and Begg's test to evaluate potential publication biases in the selected literature. As illustrated in Supplementary Figures S1-S3, the shapes of the funnel plots were symmetrical. Moreover, a Begg's test provided further statistical evidence for the absence of publication bias $(P=0.57$ for rs2107425, $P=1.00$ for rs2839698, and $P=0.62$ for rs217727).

\section{DISCUSSION}

In the current study, we performed a meta-analysis by pooling 10 studies with totals of 13,392 cases and 18,893 controls. We demonstrated that the variant $\mathrm{T}$ allele of rs2107425 exhibited a significant decreased risk for developing cancer, and the A allele of rs2839698 was associated with a significant increased cancer risk

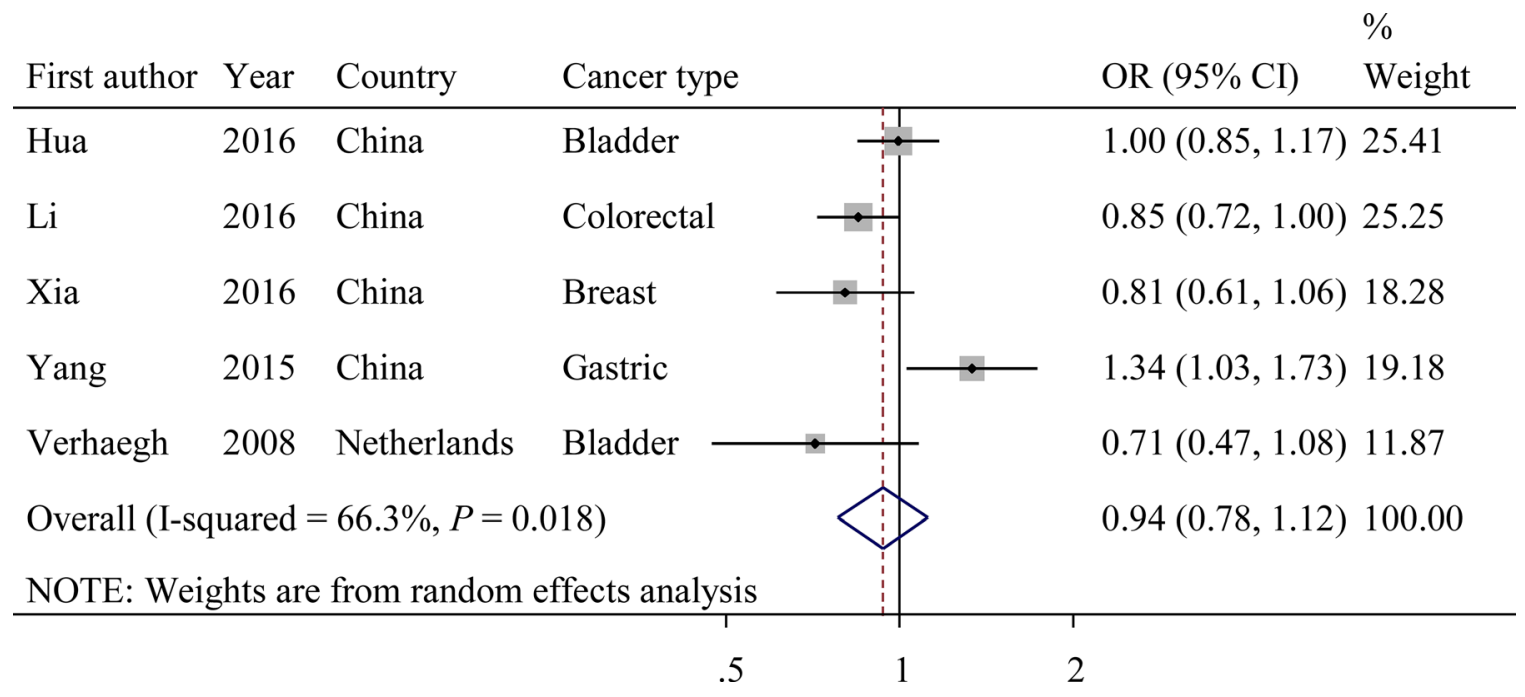

Figure 4: Forest plot of OR with $95 \%$ CI for the $H 19$ rs217727 with cancer risk under dominant model. 
in Asians, as well as a significant association with an increased risk for digestive system cancers. In contrast, the rs217727 variant allele exhibited no significant association with cancer risks.

Recently, multiple studies have reported the significant role of $H 19$ in tumourigenesis. As suggested by Chen et al. in 2016, H19 may promote gastric cancer cell migration and invasion [19], while similar results were observed in oesophageal squamous cell carcinoma cells [20, 21]. Our study indicates that $H 19$ may act as an oncogene and predict poor prognosis. Furthermore, bioinformatic analysis has shown that rs2839698 in H19 may change crucial folding structures and alter the targeted microRNAs [11]. Using the prediction of the miRNA-binding analysis website (http://bioinfo.life.hust.edu.cn/miRNASNP2/index. php), we found that rs2839698 polymorphism in H19 $3^{\prime}$ UTR may result in the loss of hsa-miR-24-1-5p and hsamiR-24-2-5p function. MiR-24 acts as a tumour suppressor and is low-expressed in various types of cancers, including colorectal, prostate and bladder cancer [22-24]. Thus, it is biologically conceivable that the loss of miR-24 function owing to a SNP rs2839698 in the 3' UTR of H19 may give rise to overexpression of $\mathrm{H} 19$ and thereby promote proliferation, migration and invasion of some cancer cells. However, experimental studies to evaluate the limits of this hypothesis are warranted, and future functional studies are required to clarify the possible mechanisms.

In this meta-analysis, we identified a marginally significant relationship of rs2839698 SNP with overall cancer risk. According to a stratified analysis, SNP rs2839698 was definitely associated with an increased risk of developing cancer for Asians (mainly Chinese patients). By contrast, among a population in the Netherlands, the same variant genotype of rs2839698 exhibited no significant association with cancer risk, and the effect value was even in the opposite direction relative to previous studies of Chinese populations. There are several possible reasons for different results between Asians and Caucasians. First, the difference may have resulted from differences in the genetic backgrounds of the studied populations. For instance, based on the HapMap data (International HapMap Project), the A allele frequency of the rs2839698 SNP is 0.28 in Asian populations $(\mathrm{CHB}+\mathrm{JPT})$ and 0.55 in European populations (CEU). Second, differences may stem from the utilization of different genotyping methods including PCR-RFLP, CRSRFLP, TaqMan and Sequenom. Third, when compared with Asian populations, the sample sizes of the Caucasian populations might not have been sufficiently large to reach a convincing conclusion concerning the association of the rs2839698 SNP with cancer risks. Additionally, the different types of cancers involved and random errors may also have been potential reasons for different findings between Asians and Caucasians.

The sensitivity analysis of rs217727 found that the result of pooling ORs was significantly changed once
Yang et al.'s study was excluded. This change could be accounted for by the 500 mixed types of gastric cancer patients (221 cardia gastric cancer patients and 279 noncardia gastric cancer patients) enrolled in Yang et al.'s study, in contrast to the stricter criteria used for recruiting patients into other studies. To account for this difference, we separated Yang et al.'s study into two parts (cardia gastric cancer and non-cardia gastric cancer), and reperformed the meta-analysis with each part considered separately. Interestingly, heterogeneity obviously decreased when Yang et al.'s study was confined to noncardia gastric carcinoma (dominant model: $P$ for heterogeneity $=0.062, I^{2}=55.4 \%$ ). However, when Yang et al.'s study was confined to cardia gastric carcinoma patients, heterogeneity was still present (dominant model: $P$ for heterogeneity $=0.043, I^{2}=59.3 \%$ ). So these conclusions should be considered cautiously.

The strength of our meta-analysis stems from systematically reviewing the relationships between three H19 polymorphisms (rs2107425, rs2839698 and rs217727) and cancer susceptibility for the first time. In addition, the well-designed search and selection methods significantly increased the statistical power of this metaanalysis. However, there are also some limitations that need to be addressed. First, significant heterogeneity between the studies was observed for the analyses of rs2107425 and rs217727. Among the 10 published studies contained in our meta-analysis, some studies were population-based, while others were hospital-based. Second, in some studies, detailed information (e.g., age, gender, smoking status, and alcohol consumption) was not provided, which further limited the stratification analyses. Moreover, if we had been able to acquire more detailed information, we would have been able to achieve more precise estimations by adjusting for other potential covariates. Finally, few studies were included in this meta-analysis, and this small sample size limits the power to detect the associations. Because the power of funnel plots and Begg's test of publication bias may also greatly constrain our analysis, our conclusions should be interpreted cautiously. Well-conducted studies with larger sample sizes are needed to further explore the cancer risks related to H19 SNPs, especially in Caucasians.

\section{MATERIALS AND METHODS}

\section{Identification and eligibility criteria of relevant studies}

A comprehensive literature search of research published before July 31, 2016 was performed using PubMed and Web of Science using the following keywords: ("H19"), ("cancer", "carcinoma", "tumor", "tumour", or "neoplasm") and ("polymorphism", "variation", "variant", or "mutation"). Only available full-text articles written in English were included in this meta-analysis. The references 
in the retrieved articles were also reviewed for possible inclusion. Studies were included if they met the following eligibility criteria: (1) case-control studies focused on the relationship between $H 19$ polymorphisms and any type of cancer, (2) at least two articles for each studied H19 polymorphism, and (3) available information concerning the genotype frequency of each included H19 SNP (i.e., rs2107425, rs2839698 or rs217727). The exclusion criteria were as follows: (1) Studies that did not focus on cancer risk, (2) did not study H19 SNPs (rs2107425, rs2839698 or rs217727), (3) did not report the relevant genotype frequency data, (4) were not published in English, or (5) non-human research was involved. Finally, a total of 10 articles containing 13,392 cases and 18,893 controls were included in this meta-analysis (Figure 1).

\section{Data extraction}

Two investigators (M.C. and L.M.) independently extracted the data and agreed on the criteria and selections. Each article was mined for the following information: year of publication; name of the first author, ethnicity and country of origin; the type of cancer studied; the numbers of cases and controls; the source of controls; genotyping platform; and genotyped SNPs. We categorized ethnicities either as Caucasian or Asian.

\section{Quality assessments of the included studies}

The methodological quality of each included study was evaluated using the Newcastle-Ottawa quality assessment scale (NOS). Using this method, each study was judged on standard criteria and subsequently categorized based on three factors: selection, comparability, and exposure. Summary scores ranging from 0 to 9 points were calculated, where higher scores indicate lower risks of bias (Supplementary Table S1).

\section{Statistical analysis}

The risk of cancer associated with each H19 polymorphism was estimated in each study using the odds ratio (OR) and its 95\% confidence interval (95\% CI). The between-study heterogeneity was examined with a chisquare-based $Q$ statistic test, with $P \leq 0.05$ considered as statistically significant. When heterogeneity between studies was absent we pooled the results using fixed-effect models. Otherwise, a random-effects model was chosen. Subsequently, we evaluated the risks of heterozygous and variant homozygous genotypes relative to the wild-type homozygous genotype, and then assessed the risks of the combined heterozygous as well as variant homozygous genotypes relative to the wild-type homozygous genotype while assuming the dominant effects of the variant allele. We performed a stratification analysis based on ethnicity (divided into Caucasians and Asians), cancer type, source of controls and case sample size. Funnel plots and Begg's test were utilized to evaluate publication bias. All analyses were performed using Stata software, version 12.0 (Stata Corporation, College Station, TX, and USA).

\section{CONCLUSIONS}

This meta-analysis provided evidence that $H 19$ rs2107425 may modify general cancer susceptibility, while rs2839698 may modify cancer susceptibility based on ethnicity and type. Beyond these conclusions, we believe further studies that incorporate subjects from different ethnic backgrounds combined with resequencing the marked regions and functional evaluations are warranted.

\section{ACKNOWLEDGMENTS AND FUNDING}

This work was funded by the National Natural Science Foundation of China (81502876), the Natural Science Research of Jiangsu Higher Education Institutions (15KJB330006), the Science and Technology Program of Nantong City (MS22015088, HS2014041) and the Doctoral Scientific Research Foundation of Nantong University (14R16). The funding sources had no role to play in the study design, the collection and interpretation of the data, writing of the report, or decision to submit this paper for publication.

\section{CONFLICTS OF INTEREST}

None.

\section{REFERENCES}

1. Siegel RL, Miller KD, Jemal A. Cancer statistics, 2016. CA Cancer J Clin. 2016; 66:7-30.

2. Chen W, Zheng R, Baade PD, Zhang S, Zeng H, Bray F, Jemal A, Yu XQ, He J. Cancer statistics in China, 2015. CA Cancer J Clin. 2016; 66:115-132.

3. Bartonicek N, Maag JL, Dinger ME. Long noncoding RNAs in cancer: mechanisms of action and technological advancements. Mol Cancer. 2016; 15:43.

4. Zhang F, Zhang L, Zhang C. Long noncoding RNAs and tumorigenesis: genetic associations, molecular mechanisms, and therapeutic strategies. Tumour Biol. 2016; 37:163-175.

5. Iyer MK, Niknafs YS, Malik R, Singhal U, Sahu A, Hosono Y, Barrette TR, Prensner JR, Evans JR, Zhao S, Poliakov A, Cao X, Dhanasekaran SM, et al. The landscape of long noncoding RNAs in the human transcriptome. Nat Genet. 2015; 47:199-208.

6. Chen T, Yang P, He ZY. Long noncoding RNA H19 can predict a poor prognosis and lymph node metastasis: a meta-analysis in human cancer. Minerva Med. 2016. 
7. Easton DF, Pooley KA, Dunning AM, Pharoah PD, Thompson D, Ballinger DG, Struewing JP, Morrison J, Field H, Luben R, Wareham N, Ahmed S, Healey CS, et al. Genome-wide association study identifies novel breast cancer susceptibility loci. Nature. 2007; 447:1087-1093.

8. Riaz M, Berns EM, Sieuwerts AM, Ruigrok-Ritstier K, de Weerd V, Groenewoud A, Uitterlinden AG, Look MP, Klijn JG, Sleijfer S, Foekens JA, Martens JW. Correlation of breast cancer susceptibility loci with patient characteristics, metastasis-free survival, and mRNA expression of the nearest genes. Breast Cancer Res Treat. 2012; 133:843-851.

9. Yang C, Tang R, Ma X, Wang Y, Luo D, Xu Z, Zhu Y, Yang L. Tag SNPs in long non-coding RNA H19 contribute to susceptibility to gastric cancer in the Chinese Han population. Oncotarget. 2015; 6:15311-15320. doi: 10.18632/oncotarget.3840.

10. Hua Q, Lv X, Gu X, Chen Y, Chu H, Du M, Gong W, Wang M, Zhang Z. Genetic variants in lncRNA H19 are associated with the risk of bladder cancer in a Chinese population. Mutagenesis. 2016.

11. Li S, Hua Y, Jin J, Wang H, Du M, Zhu L, Chu H, Zhang Z, Wang M. Association of genetic variants in lncRNA H19 with risk of colorectal cancer in a Chinese population. Oncotarget. 2016; 7:25470-7. doi: 10.18632/oncotarget.8330.

12. Xia Z, Yan R, Duan F, Song C, Wang P, Wang K. Genetic Polymorphisms in Long Noncoding RNA H19 Are Associated With Susceptibility to Breast Cancer in Chinese Population. Medicine (Baltimore). 2016; 95:e2771.

13. Gong WJ, Yin JY, Li XP, Fang C, Xiao D, Zhang W, Zhou HH, Li X, Liu ZQ. Association of well-characterized lung cancer lncRNA polymorphisms with lung cancer susceptibility and platinum-based chemotherapy response. Tumour Biol. 2016; 37:8349-8358.

14. Verhaegh GW, Verkleij L, Vermeulen SH, den Heijer M, Witjes JA, Kiemeney LA. Polymorphisms in the H19 gene and the risk of bladder cancer. Eur Urol. 2008; 54:1118-1126.

15. Butt S, Harlid S, Borgquist S, Ivarsson M, Landberg G, Dillner J, Carlson J, Manjer J. Genetic predisposition, parity, age at first childbirth and risk for breast cancer. BMC Res Notes. 2012; 5:414.

16. Barnholtz-Sloan JS, Shetty PB, Guan X, Nyante SJ, Luo J, Brennan DJ, Millikan RC. FGFR2 and other loci identified in genome-wide association studies are associated with breast cancer in African-American and younger women. Carcinogenesis. 2010; 31:1417-1423.

17. Quaye L, Tyrer J, Ramus SJ, Song H, Wozniak E, DiCioccio RA, McGuire V, Hogdall E, Hogdall C, Blaakaer J, Goode EL, Schildkraut JM, Easton DF, et al. Association between common germline genetic variation in 94 candidate genes or regions and risks of invasive epithelial ovarian cancer. PLoS One. 2009; 4:e5983.

18. Song H, Ramus SJ, Kjaer SK, DiCioccio RA, ChenevixTrench G, Pearce CL, Hogdall E, Whittemore AS, McGuire V, Hogdall C, Blaakaer J, Wu AH, Van Den Berg DJ, et al. Association between invasive ovarian cancer susceptibility and 11 best candidate SNPs from breast cancer genome-wide association study. Hum Mol Genet. 2009; 18:2297-2304.

19. Chen JS, Wang YF, Zhang XQ, Lv JM, Li Y, Liu XX, Xu TP. H19 serves as a diagnostic biomarker and up-regulation of H19 expression contributes to poor prognosis in patients with gastric cancer. Neoplasma. 2016; 63:223-230.

20. Zhou YW, Zhang H, Duan CJ, Gao Y, Cheng YD, He D, Li R, Zhang CF. miR-675-5p enhances tumorigenesis and metastasis of esophageal squamous cell carcinoma by targeting REPS2. Oncotarget. 2016; 7:30730-47. doi: 10.18632/oncotarget.8950.

21. Tan D, Wu Y, Hu L, He P, Xiong G, Bai Y, Yang K. Long noncoding RNA H19 is up-regulated in esophageal squamous cell carcinoma and promotes cell proliferation and metastasis. Dis Esophagus. 2016.

22. Gao Y, Liu Y, Du L, Li J, Qu A, Zhang X, Wang L, Wang C. Down-regulation of miR-24-3p in colorectal cancer is associated with malignant behavior. Med Oncol. 2015; 32:362.

23. Goto Y, Kojima S, Nishikawa R, Enokida H, Chiyomaru T, Kinoshita T, Nakagawa M, Naya Y, Ichikawa T, Seki N. The microRNA-23b/27b/24-1 cluster is a disease progression marker and tumor suppressor in prostate cancer. Oncotarget. 2014; 5:7748-7759. doi: 10.18632/oncotarget.2294.

24. Inoguchi S, Seki N, Chiyomaru T, Ishihara T, Matsushita R, Mataki H, Itesako T, Tatarano S, Yoshino H, Goto Y, Nishikawa R, Nakagawa M, Enokida H. Tumoursuppressive microRNA-24-1 inhibits cancer cell proliferation through targeting FOXM1 in bladder cancer. FEBS Lett. 2014; 588:3170-3179. 